\title{
Oligofuran-Benzothiadiazole Co-oligomers: Synthesis, Optoelectronic Properties and Reactivity
}

\author{
Dror Ben Abba Amiel ${ }^{a}$ (i) \\ Choongik Kim ${ }^{b}$ (D) \\ Ori Gidron* $^{*}$ (D) \\ a Institute of Chemistry, The Hebrew University of Jerusalem, Edmond J. Safra Campus, \\ Jerusalem 91904, Israel \\ b Department of Chemical and Biomolecular Engineering, Sogang University, Seoul, \\ 04107, South Korea \\ ori.gidron@mail.huji.ac.il
}

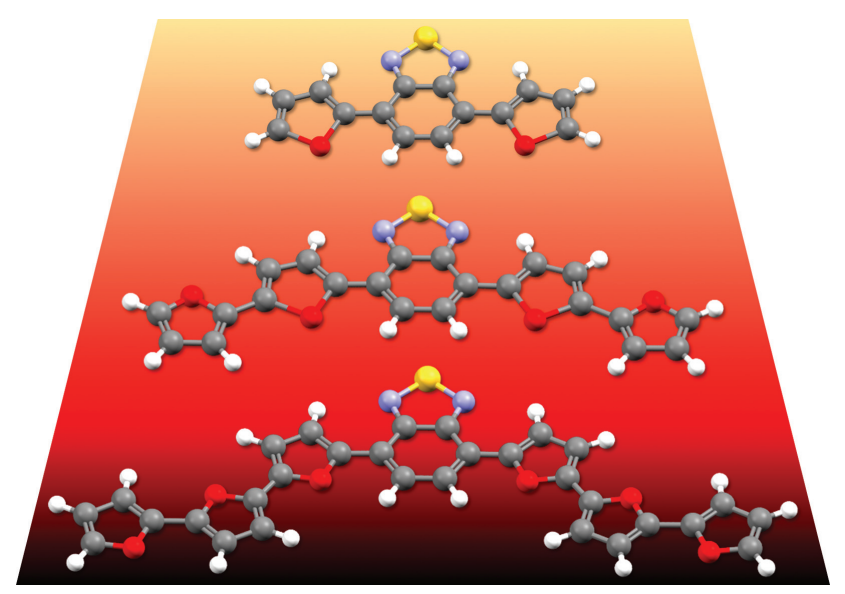

polymers, the donors are oligothiophene units. Indeed, DAD triads containing mono-, di-, or terthiophenes as donors and benzothiadiazole (BT) as the acceptor (nT-BT) have been intensively studied and demonstrate excellent performance as active materials in many of the aforementioned technologies. However, oligothiophenes are twisted from planarity, which hampers conjugation and charge delocalization and thus limits the range of thiophene-based materials suitable for such applications.

In recent years, we have introduced oligofurans $(\boldsymbol{n F}$; Scheme 1) as oligothiophene analogs that contain oxygen (rather than sulfur) as the heteroatom. ${ }^{6}$ Oligofurans tend to be more reactive than oligothiophenes (for example, they can be used as the diene in Diels-Alder reactions), ${ }^{7}$ which makes unsubstituted polyfurans generally unstable under ambient conditions, and therefore not applicable for organic electronics. ${ }^{8-11}$

Notwithstanding these drawbacks, oligofurans show a number of advantages over their thiophene analogs. (i) They are more soluble, ${ }^{4}$ which is a significant factor in their processing, (ii) their crystal packing is more efficient, ${ }^{3}$ which leads to better charge transport, and (iii) they are more rigid and planar, ${ }^{5}$ which improves their conjugation and enhances their fluorescence, both in terms of a lower Stokes shift and in terms of a higher fluorescence quantum yield $(\mathrm{QY}){ }^{3}$

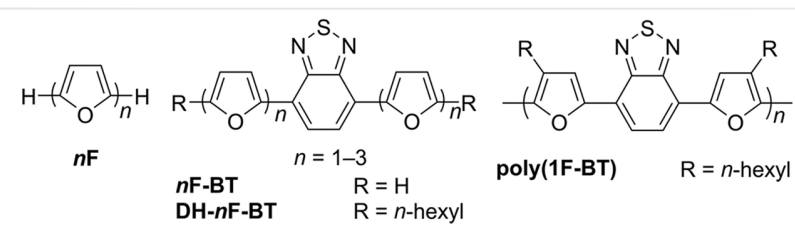

Scheme 1 Structures of nF-BT and poly(1F-BT).

\section{Introduction}

Conjugated oligomers with a donor-acceptor-donor (DAD) structure and low HOMO-LUMO gap are important electroactive and photoactive materials for applications in biochemical fluorescence technology, ${ }^{1,2}$ nonlinear optics, ${ }^{2}$ organic light-emitting diodes, ${ }^{3,4}$ organic field-effect transistors (OFETs), ${ }^{5}$ and photovoltaic cells. In many of these 
Oligofurans, such as 6F, have already been used successfully in the fabrication of OFETs, and have shown field-effect mobilities similar to those of their thiophene analogs. ${ }^{12}$ Their greater planarity and rigidity (and consequently better conjugation and charge delocalization) and their stronger fluorescence also make them promising candidates as the donor unit in DAD systems. Furthermore, as the HOMO level is ca. $0.3 \mathrm{eV}$ higher in $\boldsymbol{n F}$ compared with analogous thiophenes, combining $\boldsymbol{n F}$ donors with suitable acceptors in a DAD structure is expected to result in a lower bandgap, leading to emission in the NIR spectral region.

Recently, a donor-acceptor (DA) polymer utilizing BT as the acceptor unit and bifuran as the donor was synthesized and shown to possess excellent ambipolar mobility. ${ }^{13}$ Such polymers show great potential. However, very few have been investigated. Consequently, many questions still remain, such as the issue of the photostability of oligofurans in DA systems, which is considered their "Achilles' heel." Moreover, it is not yet clear how bandgap is affected by backbone extension. Specifically, it is not known to what extent furan units need to be extended to obtain the low bandgap required for ambipolar properties and NIR emission nor the number of units above which these properties converge.

To answer these questions, we adopted a systematic "oligomer approach." Here, we introduce a series of DAD triads with monofuran, bifuran, or terfuran as donors and BT as the acceptor (nF-BT; Scheme 1). Our combined computational and experimental investigation demonstrated that DAD systems incorporating oligofuran donors exhibit bandgaps similar to those of their thiophene analogs, which is in contrast with the trend observed for parent furans, which exhibit a ca.0.3-0.4 eV larger bandgap compared with parent thiophenes. The HOMO reaches saturation at $\mathbf{3 F - B T}$, which exhibits properties similar to those of poly(1F-BT). The $\boldsymbol{n F - B T}$ series displays fluorescence in the red-NIR spectral region, with fluorescence quantum efficiency ranging from $4 \%$ to $56 \%$. Inclusion of the BT unit significantly increases their stability; however, the compounds are still prone to photo-oxidation, and stronger acceptors or capping units should be considered in future.

\section{Results and Discussion}

To assess the contribution of oligofurans used instead of oligothiophenes as donors in DAD systems, we performed a computational investigation of the properties of $\boldsymbol{n F - B T}$ and their thiophene analogs, nT-BT, at the DFT/B3LYP-6-311G(d) level of theory. Figure 1 displays the HOMO and LUMO energies and bandgaps of $\boldsymbol{n F - B T}$ and $\boldsymbol{n T}$-BT. As the donor is extended from 1F-BT to 3F-BT, the HOMO energy increases significantly (by $0.55 \mathrm{eV}$ ), while the LUMO energy remains almost unchanged. As can be observed, unlike parent furans, which exhibit ca. 0.3-0.4 eV higher HOMO-LUMO gaps compared

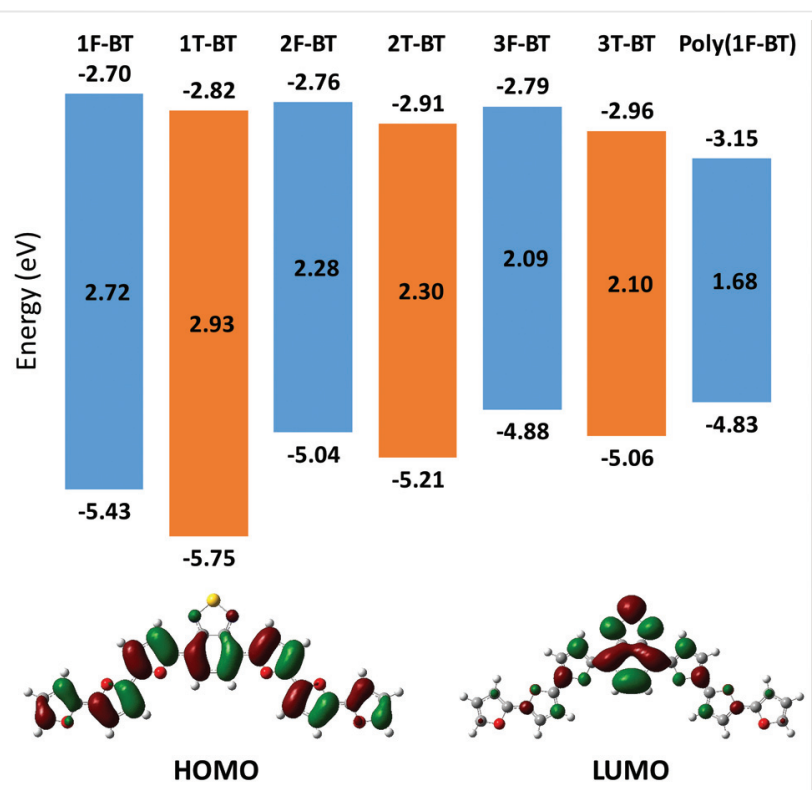

Figure 1 The calculated (B3LYP/6-311(d)) HOMO (bottom) and LUMO (top) energies (eV) and the HOMO-LUMO energy gap (center) of $\boldsymbol{n F}$-BT compounds and of the polymer poly(nF-BT). Bottom: HOMO and LUMO orbitals of 3F-BT.

with thiophenes, the HOMO-LUMO gap for $\boldsymbol{n F - B T}$ is almost identical to that of $\boldsymbol{n T}$-BT. This can be explained by the higher HOMO energy levels of oligofurans, which makes them better donor units, so increasing the efficiency of a DAD triad. In fact, a 3F-BT DAD triad exhibits a similar HOMO-LUMO gap to that of poly(1F-BT) calculated at the $\mathrm{PBC} / \mathrm{B} 3 \mathrm{LYP} / 6-311$ (d) level of theory. The increase in HOMO energy with oligomer length is due to furan ring conjugation along the chain. However, once we expand the chain to a polymer, a significant decrease in the LUMO energy is clearly visible, because of the increasing ratio of acceptors to donors. The calculated HOMO-LUMO gap for the polymer is $1.68 \mathrm{eV}$, which corresponds to a wavelength of $738 \mathrm{~nm}$, indicating that such a polymer should have an emission peak extending well within the NIR spectral range. The calculated HOMO and LUMO orbitals are separated as exemplified in Figure 1 for 3F-BT, indicating a clear charge transfer nature for the lowest energy $\left(\pi-\pi^{*}\right)$ transition.

Long conjugated systems are commonly functionalized with alkyl substituents to render them soluble. ${ }^{14}$ We therefore calculated derivatives of $\boldsymbol{n F}$-BT substituted with $n$-hexyl $(\mathrm{H})$ groups at the terminal $\alpha$-positions (DH-nF-BT). The calculated energy levels show a decrease in the HOMO-LUMO gap for all the hexyl-substituted assemblies compared with the unsubstituted parent, with a greater decrease observed for shorter oligomers. Upon alkyl substitution, both the HOMO and LUMO energy levels increase, but the increase is greater for the HOMO level, resulting in an overall decrease in the HOMO-LUMO gap in DH-nF-BT compared with $\boldsymbol{n F - B T}$. 


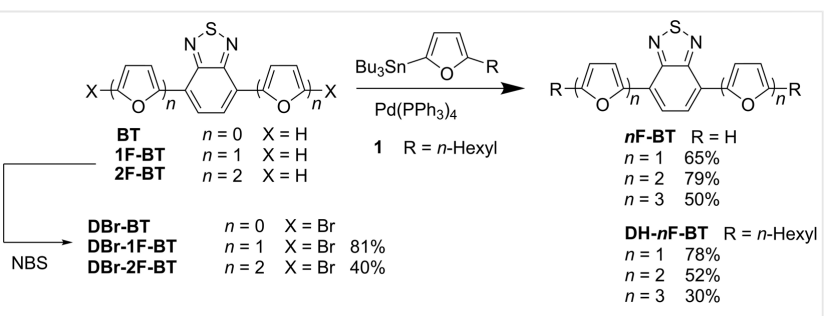

Scheme 2 Synthetic pathway for nF-BT and DH-nF-BT. NBS = Nbromosuccinimide.

For the experimental component of this study, 1F-BT was synthesized by Stille coupling of 2-tributylstannylfuran and 4,7-dibromobenzothiadiazole (DBr-BT; Scheme 2). ${ }^{15}$ The chain length was then extended via bromination with $N$ bromosuccinimide (NBS) to afford DBr-1F-BT, ${ }^{16}$ followed by additional Stille coupling to give 2F-BT as a dark red solid. ${ }^{17}$ Additional bromination using the same method yielded $\mathbf{D B r}$ 2F-BT, which was coupled again with 2-tributylstannylfuran to produce 3F-BT. However, the product identified as $\mathbf{3 F - B T}$ was almost completely insoluble, and therefore its structure could not be confirmed using NMR.

To overcome the low solubility observed for 2F-BT and 3F-BT, we introduced hexyl-substituted derivatives of $\boldsymbol{n F - B T}$ at the $\alpha$-terminal positions (DH-nF-BT; Scheme 1). In addition to increased solubility, such materials are expected to protect the reactive $\alpha$-positions in furans. ${ }^{8}$ Moreover, it was previously observed that $\alpha$-hexyl-oligofurans displayed superior fieldeffect mobility compared with the unsubstituted derivatives, which was explained by the formation of an ordered layered morphology. ${ }^{12}$ To this end, commercially available 2-hexylfuran was stannylated using $n$-butyllithium followed by the addition of tributylstannyl chloride, resulting in $\mathbf{1}$ (Scheme 2), which was then coupled with each of DBr-BT, DBr-1F-BT, and DBr-2F-BT to produce DH-nF-BT $(n=1-3)$. As expected, the hexyl-substituted products displayed better solubility, with the main effect of substitution being a bathochromic shift in their UV-vis absorption spectra.

To assess the potential of oligofuran-DAD materials for optoelectronic applications, we investigated their photophysical properties. Figure 2a displays the absorbance spectra of DH-nF-BT hexane. Unlike their thiophene analogs, the absorption spectra of DH-1F-BT and DH-2F-BT display vibronic shoulders, an indication of their rigid structure. Although the difference in the maximal absorbance wavelength changes significantly from DH-1F-BT $\left(E_{\mathrm{g}}{ }^{\mathrm{op}}=2.26 \mathrm{eV}\right.$, calculated by onset of absorption) to DH-2F-BT $\left(E_{\mathrm{g}}{ }^{\mathrm{op}}=2.05 \mathrm{eV}\right)$, it quickly converges, and DH-3F-BT $\left(E_{\mathrm{g}}{ }^{\text {op }}=1.98 \mathrm{eV}\right)$ is only slightly bathochromically shifted compared with DH-2F-BT. Whereas parent oligofurans always display a larger bandgap (by ca. $0.3 \mathrm{eV}$ ) compared with parent oligothiophenes, $\boldsymbol{n F - B T}$ displays bandgaps very similar to those of $\boldsymbol{n T}$-BT, so confirming the abovementioned trend in calculated HOMO and LUMO energies. Attempts to perform
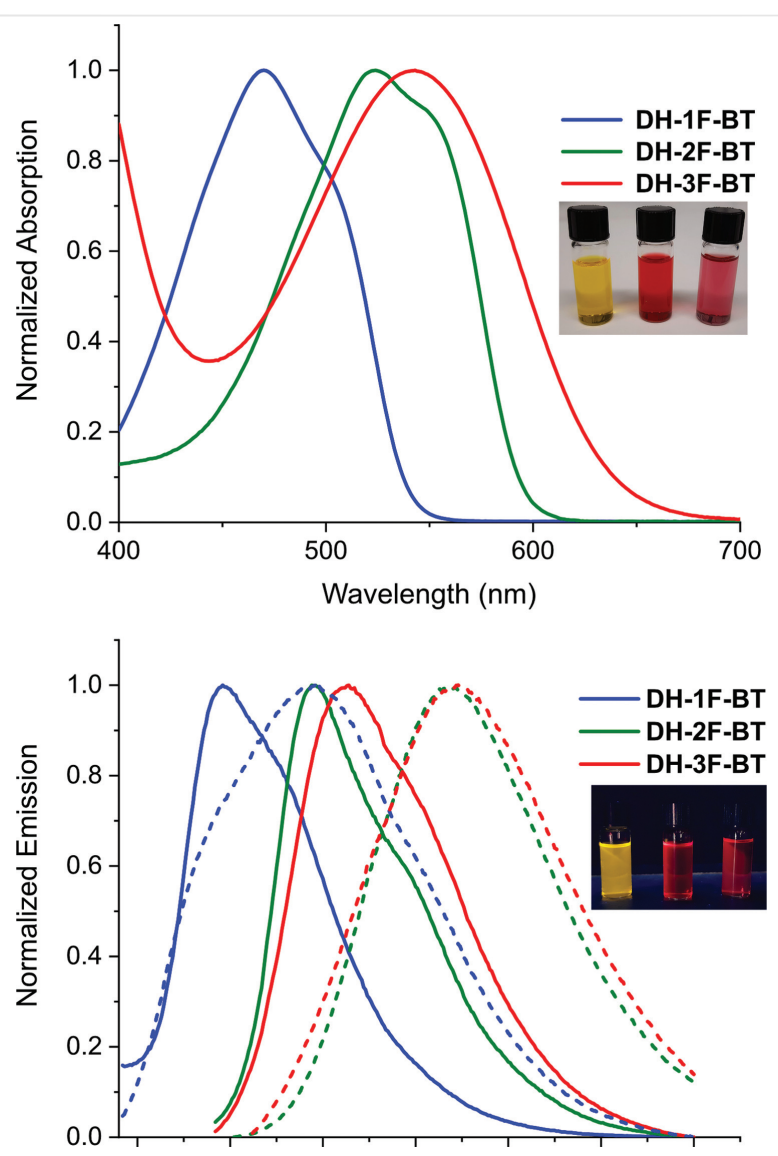

Figure 2 (a) Absorption in DCM. (b) Emission in DCM (dashed) and hexane (solid) of DH-1F-BT, DH-2F-BT, and DH-3F-BT. Insets: vials containing solutions of DH-nF-BT in DCM under ambient light (top) and under 365 nm UV light (bottom).

cyclic voltammetry did not result in clear reversible peaks for the longer DAD system, which is attributed to their low solubility.

Substitution with hexyl groups results in bathochromic shifts, observed in both the absorption and emission spectra (Figure S23, see the Supporting Information, SI). The shift ranges from $0.16 \mathrm{eV}$ for 1F-BT to $0.08 \mathrm{eV}$ for 2F-BT, while for 3F-BT there is no observable shift. This observation is in line with our DFT (density functional theory) calculations, which showed that the energy increase was larger for the HOMO compared with the LUMO, resulting in an overall decrease in the HOMO-LUMO energy gap. The calculated HOMO-LUMO gaps are $0.14 \mathrm{eV}$ for 1F-BT and $0.07 \mathrm{eV}$ for 2F-BT. In contrast with the abovementioned monomer and dimer, the hexyl-substituted terfuran DAD triad is slightly blue-shifted in comparison with the non-substituted one.

The fluorescence of the $\boldsymbol{n F - B T}$ series is strongly dependent on solvent polarity, as expected for a DAD system, with a solvatochromic shift $\left(\lambda_{\mathrm{em}}\right)$ of $0.231 \mathrm{eV}$ (from $594 \mathrm{~nm}$ to $668 \mathrm{~nm}$ ) observed for 2F-BT (Figure 2b). 


\begin{tabular}{|c|c|c|c|c|}
\hline Compound & Hexane & DCM & THF & 1,4-Dioxane \\
\hline 1F-BT & 52 & 29 & 42 & 37 \\
\hline DH-1F-BT & 54 & 25 & - & 30 \\
\hline 2F-BT & 49 & 28 & 19 & 17 \\
\hline DH-2F-BT & 37 & 7 & - & 18 \\
\hline DH-3F-BT & 23 & 4 & - & - \\
\hline
\end{tabular}

Fluorescence quantum efficiency (QY) in DCM ranges from $5 \%$ for DH-3F-BT to $29 \%$ for $\mathbf{1 F - B T}$ (Table 1). As expected, QY increases in a non-polar solvent (hexane) and reaches up to 54\% (for 1F-BT). Although these values are significantly lower than for the parent furans (which reach a value of $80 \%$ for $\mathbf{4 F}$ ), they are relatively high for DAD systems, which suggests that $\boldsymbol{n} \mathbf{F}-\mathbf{B T}$ series members have potential as active materials for light-emitting devices. In this respect, 2F-BT is potentially interesting as an NIR-emitting material, with QY of $28 \%$ in the NIR spectral range, while 3F-BT shows no advantage over 2F-BT as an NIR emitter. Our data show that bifuran is an ideal candidate for donor units in DAD systems, as no significant advantage is seen with further extension to terfuran, which significantly reduces the solubility. The fluorescence lifetime for DH-nF-BT is in the range of 4.2-9.2 ns (Table 2), which is slow compared with parent furans, as expected for DA systems. Overall, DAD triads of oligofurans show similar photophysical properties as their oligothiophene analogs, with the advantage of increased solubility for the former.

To assess the properties of oligofuran-containing DA polymers, we synthesized a polymer consisting of alternating bifuran-BT units (poly(1F-BT); Scheme 1). Similar polymers can be prepared electrochemically, ${ }^{22}$ but they are too insoluble to be prepared chemically. To overcome this, we used furan units substituted with $n$-hexyl at the $\beta$-positions. The polymer was synthesized using Stille polymerization of 3,3'-dihexyl bifuran and BT, and was found to be soluble

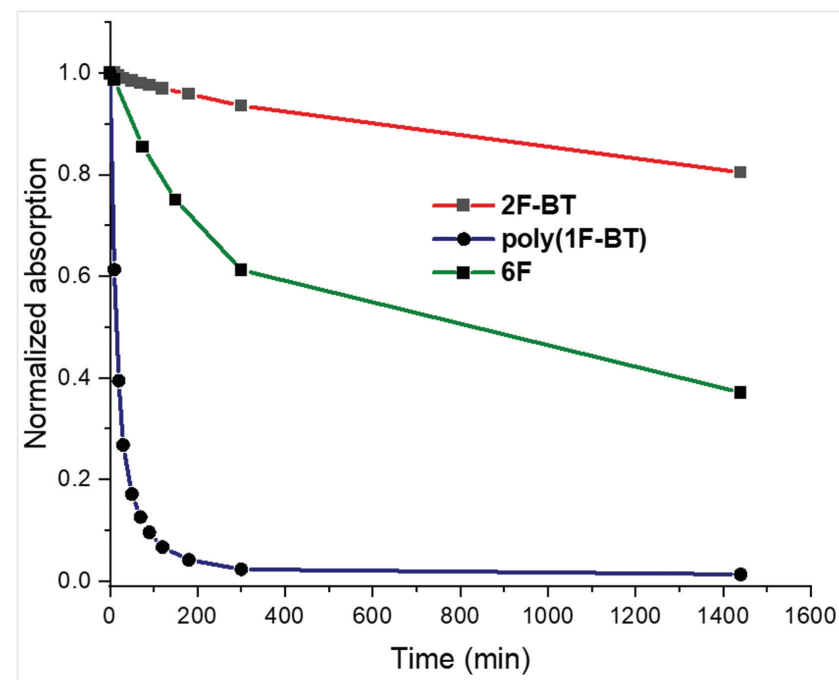

Figure 3 Photo-oxidation of 6F, 2F-BT, and poly(1F-BT) solutions in DCM vs. time.

in DCM. The polymer displayed similar absorption pattern to 3F-BT, with slightly bathochromically shifted absorption (556 nm) and emission (689 nm, Figure S22, see SI). However, the electron-withdrawing BT units provided insufficient stabilization, and the polymer rapidly photo-oxidized under ambient conditions (Figure 3). As we have previously prepared stable polyfurans consisting of bifuranimiderepeating units, ${ }^{23}$ we conclude that substitution of furans with electron-withdrawing groups at the $\beta$ (rather than $\alpha$ ) positions is necessary for their inclusion in stable polymers.

We recently found that capping furans [in an acceptordonor-acceptor (ADA) triad] or substituting them at the $\beta$ position with electron-withdrawing groups can significantly increase their stability toward photo-oxidation. ${ }^{24}$ However, the photostability of DAD assemblies with terminal oligofurans remains unknown. We therefore compared the stabilities of $\boldsymbol{n F - B T}$ and parent furans using unsubstituted $\mathbf{6 F}$ as a benchmark for photo-oxidation. Figure 3 shows that $\mathbf{6 F}$ undergoes relatively rapid photo-oxidation, with absorption

Table 2 Photophysical properties of $\mathbf{n F - B T}$ and $\mathbf{D H}-\boldsymbol{n T} \mathbf{T}-\mathbf{B T}$ in DCM. The values for the corresponding thiophene analogs are given in parentheses

\begin{tabular}{|c|c|c|c|c|c|c|c|c|c|}
\hline Compound & $\lambda_{\mathrm{abs}}(\mathrm{nm})$ & $\lambda_{\mathrm{em}}(\mathrm{nm})$ & $E_{\mathrm{g}}^{\mathrm{op}}(\mathrm{eV})^{\mathrm{a}}$ & $\begin{array}{l}\text { Stokes } \\
\text { shift (eV) }\end{array}$ & QY (\%) & $\begin{array}{l}\text { Lifetime } \\
\text { (ns) }\end{array}$ & $\begin{array}{l}\text { HOMO (eV) } \\
\text { (calc.) }^{\mathrm{b}}\end{array}$ & $\begin{array}{l}\operatorname{LUMO}(\mathrm{eV}) \\
\text { (calc.) }^{\mathrm{b}}\end{array}$ & $\begin{array}{l}E_{g}(\mathrm{eV}) \\
(\text { calc. })^{b}\end{array}$ \\
\hline 1F-BT & $450(445)^{18}$ & $564(577)^{14}$ & $2.42(2.40)^{19}$ & 0.56 & 29 & - & -5.43 & -2.70 & 2.73 \\
\hline DH-1F-BT & $474(469)^{20}$ & $589(603)^{20}$ & 2.26 & 0.51 & 25 & 9.2 & -5.13 & -2.54 & 2.59 \\
\hline 2F-BT & $512(502)^{21}$ & $649(611)^{19}$ & $2.13(2.20)^{21}$ & 0.51 & 28 & - & -5.04 & -2.76 & 2.28 \\
\hline DH-2F-BT & $527(510)^{14}$ & $668(656)^{14}$ & $2.05(2.07)^{14}$ & 0.50 & 7 & 4.2 & -4.86 & -2.65 & 2.21 \\
\hline 3F-BT & 544 & 676 & 1.96 & 0.45 & - & - & -4.88 & -2.79 & 2.09 \\
\hline DH-3F-BT & $543(533)^{14}$ & $673(672)^{14}$ & $1.98(1.95)^{14}$ & 0.44 & 4 & 4.2 & -4.75 & -2.72 & 2.03 \\
\hline poly(1F-BT) & 556 & 689 & 1.80 & 0.43 & - & - & -4.83 & -3.15 & 1.68 \\
\hline
\end{tabular}

${ }^{\mathrm{a} C a l c u l a t e d ~ f r o m ~ t h e ~ a b s o r p t i o n ~ o n s e t . ~}$

${ }^{b}$ Calculated at the DFT/B3LYP-6-311G(d) level of theory. 
decreasing by $15 \%$ after $75 \mathrm{~min}$ and by $63 \%$ after $24 \mathrm{~h}$ (1440 min) under ambient conditions. In contrast, the decomposition of 2F-BT is considerably slower, with a $6 \%$ reduction in absorption after 6 h and only a $15 \%$ reduction after $24 \mathrm{~h}$. These results indicate that the electron-withdrawing properties of BT increase the overall stability of the 2F-BT assembly toward photo-oxidation, as its decomposition rate is significantly slower than that for $\mathbf{6 F}$. However, the presence of BT does not fully stabilize the molecule, as photo-oxidation is observed after longer periods.

\section{Conclusions}

In this work, we investigated the $\boldsymbol{n F - B T}$ series of DAD systems in which oligofurans serve as donors. We found that their optical bandgap is similar to that of their thiophene analogs, whereas their HOMO-LUMO gap decreases more rapidly, owing to the better conjugation and donor properties of oligofurans. Fluorescence in the NIR spectral region is observed for systems in which bifuran is the donor (2F-BT), with only a small decrease in the emission maxima for terfuran (3F-BT). However, quantum efficiency is significantly lower in 3F-BT, making 2F-BT an ideal candidate in the series for NIR emitter. The addition of a BT acceptor unit significantly increases the photostability of $\boldsymbol{n F - B T}$ compared with parent furans. However, $\boldsymbol{n F - B T}$ are less stable compared with ADA oligofurans and with oligofurans substituted with electron-withdrawing groups at their $\beta$-positions. This leads to the conclusion that while the inclusion of bifuran induces strong fluorescence in DA oligomers and polymers, either stronger acceptors or capping units should be considered when designing oligofuran-based systems.

\section{Funding Information}

This work was supported by a cooperative grant from the Ministry of Science and Technology (MOST) of Israel and the Ministry of Science, ICP and Future Planning (MICP) of the Republic of Korea.

\section{Supporting Information}

Supporting Information for this article is available online at https://doi.org/10.1055/s-0041-1729853.

\section{References and Notes}

(1) Kudo, K.; Tanaka, S.; Iizuka, M.; Nakamura, M. Thin Solid Films 2003, 438, 330.

(2) Capelli, R.; Toffanin, S.; Generali, G.; Usta, H.; Facchetti, A.; Muccini, M. Nat. Mater. 2010, 9, 496.
(3) Gidron, O.; Varsano, N.; Shimon, L. J. W.; Leitus, G.; Bendikov, M. Chem. Commun. 2013, 49, 6256.

(4) Zhen, S.; Lu, B.; Xu, J.; Zhang, S.; Li, Y. RSC Adv. 2014, 4, 14001.

(5) Politis, J. K.; Nemes, J. C.; Curtis, M. D. J. Am. Chem. Soc. 2001, 123, 2537.

(6) Gidron, O.; Diskin-Posner, Y.; Bendikov, M. J. Am. Chem. Soc. 2010, 132, 2148.

(7) Gadakh, S.; Shimon, L. J. W.; Gidron, O. Angew. Chem. 2017, 129, 13789.

(8) Glenis, S.; Benz, M.; LeGoff, E.; Schindler, J. L.; Kannewurf, C. R.; Kanatzidis, M. G. J. Am. Chem. Soc. 1993, 115, 12519.

(9) Bakhshi, A. K.; Ladik, J.; Seel, M. Phys. Rev. B: Condens. Matter 1987, 35, 704.

(10) González-Tejera, M. J.; de la Blanca, E. S.; Carrillo, I. Synth. Met. 2008, 158, 165.

(11) Barak, A. H.; de Ruiter, G.; Lahav, M.; Sharma, S.; Gidron, O.; Evmenenko, G.; Dutta, P.; Bendikov, M.; van der Boom, M. E. Chem. Eur. J. 2013, 19, 8821.

(12) Gidron, O.; Dadvand, A.; Sheynin, Y.; Bendikov, M.; Perepichka, D. F. Chem. Commun. 2011, 47, 1976.

(13) Shi, S.; Wang, H.; Uddin, M. A.; Yang, K.; Su, M.; Bianchi, L.; Chen, P.; Cheng, X.; Guo, H.; Zhang, S.; Woo, H. Y.; Guo, X. Chem. Mater. 2019, 31, 1808.

(14) Melucci, M.; Favaretto, L.; Zanelli, A.; Cavallini, M.; Bongini, A.; Maccagnani, P.; Ostoja, P.; Derue, G.; Lazzaroni, R.; Barbarella, G. Adv. Funct. Mater. 2010, 20, 445.

(15) Kawabata, K.; Takeguchi, M.; Goto, H. Macromol. 2013, 46, 2078.

(16) Synthesis of DBr-1F-BT. 1F-BT (3.4 mmol, $904 \mathrm{mg}$ ) was dissolved in $50 \mathrm{~mL}$ of chloroform in a $100 \mathrm{~mL}$ round-bottom flask. Added some sodium bicarbonate and stirred under a $\mathrm{CaCl}_{2}$ drying tube in an ice bath. After $20 \mathrm{~min}$ added NBS (6.7 mmol, $1.2 \mathrm{~g}$ ) and stirred for $1 \mathrm{~h}$. The product formed as a bright orange precipitate. The solvent was evaporated almost completely, and the crude product was washed with sodium bicarbonate solution, hexane, and methanol on a sintered glass funnel to give DBr-1F-BT (1.163 g, 81\%) as a bright orange solid. ${ }^{1} \mathrm{H}$ NMR (500 MHz, chloroform-d) $\delta 8.00$ (s, $2 \mathrm{H}, \mathrm{H}-\mathrm{C} 6), \quad 7.65$ (d, $J=3.5 \mathrm{~Hz}, 2 \mathrm{H}, \mathrm{H}-\mathrm{C} 3$ ), 6.55 (d, $J=3.5 \mathrm{~Hz}, 2 \mathrm{H}, \mathrm{H}-\mathrm{C} 4$ ) ppm. ${ }^{13} \mathrm{C}$ NMR (126 MHz, chloroform-d) $\delta 152.16$ (C8), 150.94 (C2), 123.53 (C6), 123.39 (C5), 121.09 (C7), 114.95 (C4), 114.52 (C3) ppm. MS (MALDI) $m / z$ (\%): $426.8565\left(100,[\mathrm{M}+\mathrm{H}]^{+}\right)$calcd. for $\mathrm{C}_{14} \mathrm{H}_{6} \mathrm{Br}_{2} \mathrm{O}_{2} \mathrm{~N}_{2} \mathrm{~S}: 425.8569$.

(17) Synthesis of 2F-BT. Dry toluene $(50 \mathrm{~mL})$ was placed in a $250 \mathrm{~mL}$ oven-dried two-necked round-bottom flask and bubbled with argon while heating up to $90{ }^{\circ} \mathrm{C}$. Added DBr-1F-BT $(2.7 \mathrm{mmol}$, $1.163 \mathrm{~g})$ and 2-tributylstannyl furan $(5.5 \mathrm{mmol}, 1.7 \mathrm{~mL})$. Bubbled argon for $15 \mathrm{~min}$, then added tetrakis(triphenylphosphine) palladium $(0)(342 \mu \mathrm{mol}, 395 \mathrm{mg})$, bubbled with argon for 15 more minutes, and left to stir under argon at $90{ }^{\circ} \mathrm{C}$. After $5 \mathrm{~h}$, following an evaluation of reaction progress with TLC, tetrakis (triphenylphosphine)palladium(0) (222 $\mu \mathrm{mol}, 257 \mathrm{mg}$ ) was added. The solution was bubbled with argon for $10 \mathrm{~min}$ and then left to stir under argon at $90{ }^{\circ} \mathrm{C}$ overnight. Next morning another TLC was done, and then the solvent was evaporated and the crude product was dissolved in DCM and filtered on celite, then washed with brine. Organic phase was dried (MgSO4) and evaporated. The resulting solid was purified by flash chromatography ( $\mathrm{SiO}_{2}$, DCM:hexane 6:5) to give $\mathbf{2 F - B T}$ (864 mg, 79\%) as a dark red solid. ${ }^{1} \mathrm{H}$ NMR $(500 \mathrm{MHz}$, Chloroform-d $8.12(\mathrm{~s}, 2 \mathrm{H}$, H-C6), 7.79 (dd, $J=3.5,0.6 \mathrm{~Hz}, 2 \mathrm{H}, \mathrm{H}-\mathrm{C} 5$ ), 7.49 (dd, $J=1.8$, $0.8 \mathrm{~Hz}, 2 \mathrm{H}, \mathrm{H}-\mathrm{C} 3), 6.80$ (d, $\left.J=3.5 \mathrm{~Hz}, 2 \mathrm{H}, \mathrm{H}-\mathrm{Cl}^{\prime}\right), 6.75$ (d, $2 \mathrm{H}, \mathrm{H}-$ C $\left.3^{\prime}\right), 6.54(\mathrm{dd}, J=3.4,1.8 \mathrm{~Hz}, 2 \mathrm{H}, \mathrm{H}-\mathrm{C} 4) \mathrm{ppm} .{ }^{13} \mathrm{C}$ NMR 
(126 MHz, Chloroform-d) $\delta 151.51$ (C8), 149.55 (C5'), 146.77 (C2), 146.51 (C2'), 142.51 (C5), 123.40 (C6), 121.38 (C7), 114.50 (C4'), 111.83 (C4), 108.41 (C3'), 106.30 (C3) ppm. HR-ESI-MS m/z (\%): $400.0582\left(100,[\mathrm{M}]^{+}\right)$calcd. for $\mathrm{C}_{22} \mathrm{H}_{12} \mathrm{O}_{4} \mathrm{~N}_{2} \mathrm{~S}: 400.0512$.

(18) Canola, S.; Mardegan, L.; Bergamini, G.; Villa, M.; Acocella, A.; Zangoli, M.; Ravotto, L.; Vinogradov, S. A.; Di Maria, F.; Ceroni, P.; Negri, F. Photochem. Photobiol. Sci. 2019, 18, 2180.

(19) Cansu-Ergun, E. G.; Akbayrak, M.; Akdag, A.; Önal, A. M. J. Electrochem. Soc. 2016, 163, G153.
(20) Calascibetta, A. M.; Mattiello, S.; Sanzone, A.; Facchinetti, I.; Sassi, M.; Beverina, L. Molecules 2020, 25, 3717.

(21) Jeon, Y.; Kim, T.-M.; Kim, J.-J.; Hong,J.-I. New J.Chem. 2015, 39, 9591.

(22) İçli-Özkut, M.; İpek, H.; Karabay, B.; Cihaner, A.; Önal, A. M. Polym. Chem. 2013, 4, 2457.

(23) Mulay, S. V.; Bogoslavky, B.; Galanti, I.; Galun, E.; Gidron, O. J. Mater. Chem. C 2018, 6, 11951.

(24) Yakir, H. R.; Shimon, L. J. W.; Gidron, O. Helv. Chim. Acta 2019, 102, e1900027. 